\title{
PROKLA-Redaktion
}

\section{Editorial: Gesellschaftstheorie III: Kontroversen}

Wenn sie nicht gerade zu denjenigen gehören, die Richard Wagner als umstürzenden Neuerer der klassischen Musiktraditionen schätzen, so dürfte der 200. Geburtstag des Meisters die Mehrzahl der PROKLALeser/innen eher kalt lassen. Sie verbinden damit vermutlich am ehesten Bilder der politischen, wirtschaftlichen und gesellschaftlichen Elite der Bundesrepublik, die im August nach Bayreuth wallt, um sich dort den Klängen der Tetralogie Der Ring des Nibelungen hinzugeben, vielleicht aber auch nur, um in der Öffentlichkeit den Eindruck der Kulturbeflissenheit zu erwecken, und in den Pausen an Stehtischen teuren Sekt zu trinken.

Doch der 1813 geborene Wagner war nicht nur ein Zeitgenosse des 1818 geborenen Karl Marx (sie starben sogar im selben Jahr, 1883), beide nahmen auf unterschiedliche Weise Anteil an der Revolution von 1848 , und wenn für Marx die Berichte über Arbeit und Leben der englischen Arbeiterschaft eine wichtige Grundlage für seine Überlegungen zum Los der Arbeiterklasse im Kapitalismus wurden, so weiß man aus den Tagebüchern von Wagners zweiter Frau Cosima, dass dieser von einem EnglandBesuch ähnliche Eindrücke mitbrachte, die sich insbesondere im Rheingold, dem ersten Teil des Rings, niederschlugen: „Auf der Heimfahrt von Greenwich entgeht ihm nicht der Eindruck von 'Nibelheim, Weltherrschaft, Tätigkeit, Arbeit' und von dem 'Druck des Dampfes', der überall den
'Traum Alberichs' erfüllt hat." (Holland 1990: 535) Wagners mythologische Geschichte von der Jagd nach dem Gold, von Liebeshändel, Betrug, Verrat, Mord und Totschlag, von Herrschaft und Untergang der Herrschenden ist von vielen Beobachtern immer schon als Parabel auf den Kapitalismus des 19. Jahrhunderts interpretiert worden, so auch von Franz Wilhelm Beidler, dem ersten Enkelsohn Wagners: „Die komplizierten Schachtanlagen und Hüttenwerke des Ruhrgebiets etwa vereinfachen sich zu den Werkstätten Nibelheims, die Anonymität des Kapitals, die Unsicherheit des Aktionärs enthüllt sich im verschleierten Tarnhelm. Die dämonische Kraft des Ringes, d.h. des kapitalistischen Macht- und Profitstrebens, durchdringt alle Beziehungen, löst alle Bindungen, Rechte und Sitten auf. Die von altersher herrschenden Gewalten - hier heißen sie Götter - verstricken sich im kapitalistischen Gestrüpp, und die Welt wartet auf den Menschen. Auf den Menschen, der durch Verzicht auf Besitz und Gewinn die Kraft zur befreienden Tat findet und Götter und Zwerge ablöst." (zit. nach Borchmeyer 2002: 523) Dabei ist allerdings nicht zu übersehen, dass sich Wagners kapitalismuskritischer Impetus oftmals auch mit antisemitischen und nationalistischen Vorstellungen verband, die in der Schrift Das Judentum in der Musik gipfelten.

Die Deutung des Rheingold als Sinnbild des Kapitalismus des 19. Jahrhunderts war nicht unumstritten. Als 1976 das 
hundertjährige Jubiläum der ersten Bayreuther Festspiele, der "Jahrhundertring", anstand, griff der Regisseur Patrice Chéreau sie auf. Er befreite das Werk von weihevoller Entrücktheit und jenseitigem Schwulst, holte Wagner ins Diesseits der Industriegesellschaft zurück und ließ die Rheintöchter ihre Erzählung über die Macht des Goldes vor einem Staudamm singen, die (auf Kredit gebaute) Götterburg Walhall als gründerzeitlichen Prachtbau erstehen und Siegfrieds Schwert Notung nicht in einer traulichen mittelalterlichen Schmiede, sondern im Hochofen schmieden. Das Ergebnis war ein ungeheurer Eklat, bei dem es zu Buhrufen und Handgreiflichkeiten kam, die Alt-Wagnerianer Trillerpfeifen verteilten und ebenso stabreimend wie empört auf Flugblättern „Werkschutz für Wotan“ verlangten (Der Bayreuther Jahrhundertring).

Marx selbst hielt nicht viel von Richard Wagner, den er in einem Brief abfällig als „neudeutsch-preußischen Reichsmusikanten" bezeichnete, und es ist auch nicht bekannt, dass Wagner sich umgekehrt jemals positiv zu Marx geäußert hätte. Dennoch können offenbar Verbindungen zwischen ihnen ausgemacht werden, so wenn Dietmar Holland die Gesellschaftskritik und die Suche nach Erlösung im Ring als Gegenstück zu Marxens Kritik der politischen Ökonomie und der Vision einer Aufhebung der Entfremdung sieht (Holland 1990: 535, vgl. zu Wagner und Marx auch Jäger 2013 ). Gemeinsam ist beiden ein umfassender Anspruch, der die Totalität der Gesellschaft umgreift. Marx ging es nicht allein um die Mechanismen der Ökonomie, Wagner nicht allein um die Dynamik der Leidenschaften - und genau darin liegt gleichfalls ein wiederkehrendes Anliegen der Texte zu Gesellschaftstheorie und Gesellschaftskritik, die in den letzten Jahren in der PROKLA veröffentlicht wurden, insbesondere in den Heften Gesellschaftstheorie nach Marx und Foucault (PROKLA 151/2008), Sozialismus? (155/2009), Marx! (159/2010), Gesellschaftstheorie im Anschluss an Marx (165/2011) und zuletzt Perspektiven der Gesellschaftskritik heute (167/2012).

Theoriediskussionen bilden gewissermaßen das „Kerngeschäft" der PROKLA, doch haben sich ihre Schwerpunkte im Lauf des mehr als vierzigjährigen Bestehens der Zeitschrift geändert: Zeit für eine kurze Zwischenbilanz. Bei diesen Themen gibt es in den vergangenen Jahrzehnten so etwas wie Stammgäste, die immer wieder in Erscheinung traten. Andere Themen hatten nur kurze Auftritte und schienen eher freundlich geduldete als besonders willkommene Gäste darzustellen. Und schließlich machten in den letzten Jahren auch eine Reihe von ganz neuen, bisher kaum beachteten Themen ihre Aufwartung.

Zu den Stammthemen der PROKLA gehören solche, die Theorien zur Entwicklung des Kapitalismus diskutieren, insbesondere Krisentheorien. Wiederholt gab es Debatten zur Geschichte der Krisentheorien und Auseinandersetzungen um Überakkumulations- oder Unterkonsumtionstheorien (PROKLA 14-15/1974, 22/1976, 30/1978, 32/1978, 53/1983). Unter dem Titel Krise der Ökonomie - Versagen der Krisentheorie? (57/1984) wurden ebenfalls Fragen der Profitratenentwicklung oder des ProfitSqueeze aufgeworfen. Seither hat sich der Schwerpunkt weg von diesen grundsätzlichen Fragen verschoben. Zwar wurden vielfach Fallstudien zu Krisen in einzelnen Ländern vorgelegt, ansonsten aber eher praktisch-politische Kriseninterventionen thematisiert wie in PROKLA 82/1991 zu Markt und Demokratie oder aber zu Fragen keynesianischer Politik wie beim Heftthema Marx, Keynes und der globalisierte Kapitalismus (123/2001). In neuerer Zeit wurden wirtschaftswissenschaftliche 
Theorien, insbesondere die nach wie vor hegemoniale Neoklassik verstärkt aufgegriffen, so in Kritik der Wirtschaftswissenschaften (164/2011). Eng verbunden mit diesen Diskussionen war immer wieder die Ebene des Weltmarkts, die in früheren Zeiten meist als Frage nach dem „ungleichen Tausch“ oder der ,internationalen Durchschnittsprofitrate“ gestellt wurde (PROKLA 6/1973, 8-9/1973, 60/1985). Der Begriff Imperialismus wurde erst dann intensiv diskutiert, als man ihn anderswo bereits für überholt hielt, so in Imperialistische Globalisierung (133/2003, auch in 159/2010).

Ebenfalls zu den Kernthemen der Zeitschrift gehören solche zu politischen Fragen, nach dem Staat, insbesondere dem Sozialstaat, nach Herrschaft und Demokratie, doch zeigen sich hier im Lauf der Jahre deutliche Verschiebungen. In früheren Zeiten dominierten auch hier grundsätzliche Überlegungen, wie solche zur Sozialstaatsillusion (Sonderheft 1/1971) oder zur Frage der „Ableitung“ des bürgerlichen Staates (PROKLA7/1973, 14-15/1974), außerdem wurde die Auseinandersetzung mit damals populären Ansätzen wie der Theorie des staatsmonopolistischen Kapitalismus geführt (8-9/1973). In neuerer Zeit wurde der Fokus dann weg von der nationalen auf die globale Ebene ausgedehnt, so auf die $R e$ Regulierungder Weltwirtschaft (118/2000) oder auf die Internationalisierung des Staates (147/2007).

Zentral blieb über die Jahrzehnte hinweg schließlich der explizite Bezug auf Marx, in den Anfängen vor allem als Debatte über unterschiedliche Marx-Interpretationen, so etwa bei der Diskussion über die Ergebnisse des Projekts Klassenanalyse (PROKLA 10/1973). Dieser Bezug schien in späteren Jahren jedoch weder selbstverständlich noch fraglos, und so gab es in PROKLA 72/1988 erstmals ein
Themenheft zu Marxismus obne Marx, dem seither, auch in Auseinandersetzung mit postmarxistischen Ansätzen, die bereits genannten Hefte Gesellschaftstheorie nach Marx und Foucault (151/2008) und Marx! (159/2010) folgten. Bei all dem kann erstaunen, dass es das für Marx so wichtige Thema der Klassen auf theoretischer Ebene bisher nur ein einziges Mal zum Rang eines Heftthemas brachte, Klassen und Herrschaft (PROKLA 58/1985), und zwar in einer Zeitschrift, deren Akronym PROKLA ursprünglich vor allem die Probleme des Klassenkampfs ansprechen sollte ... Die PROKLA wird diesen Mangel demnächst ansatzweise beheben: für 2014 ist ein Heft zu Klassen und Klassentheorien geplant.

Andere Themen wurden nur gelegentlich behandelt. Eines davon betrifft die Ökologie, die nur in größeren zeitlichen Abständen in den Mittelpunkt gerückt wurde, dann aber auch gleich als Heftthema: Ökologie und Marxismus (PROKLA34/1979), Ökologie und Ökonomie (67/1987), Ökologie in der Krise?(156/2009). Nicht zuletzt wurden hier auch Ansätze einer Koppelung von natur- und gesellschaftswissenschaftlichen Theorien diskutiert (PROKLA 67/1987, $159 ; 160 / 2010$ und 165/2011). Ein weiteres, eher seltenes Thema ist der Sozialismus. Zwar erfolgte bereits in den 1970er Jahren eine Auseinandersetzung mit Herrschaft in "nachkapitalistischen“ Gesellschaften (PROKLA 22/1976) und Rudolf Bahros Anatomie des real existierenden Sozialismus (31/1978), und nach den dramatischen politischen Umwälzungen von 1989/1990 wurde Auf der Suche nach dem verlorenen Sozialismus zum Heftthema (78/1990). Doch dann wurde das Thema (zweifellos nicht nur in $\operatorname{der}$ PROKLA) erst einmal beiseite gelegt. Es bedurfte fast zweier Jahrzehnte, bis das bereits weiter oben genannte Heft Sozialismus? (155/2009) erschien. 
Eine lange Zeit eher schattenhafte Existenz führte auch das Thema Geschlechterverhältnisse, das zunächst, wenn überhaupt, im Zusammenhang mit Frauenarbeit diskutiert wurde, so in einzelnen Aufsätzen als „Arbeit der Mütter“ (PROKLA 22/1976) oder als „Hausarbeit“ (33/1978), schließlich als Heftschwerpunkt Frauen in der Ökonomie (93/1993), wo auch erstmals der Ansatz der sozialen Konstruktionen von Weiblichkeit und Männlichkeit zum Thema wurde. In den letzten Jahren wurden Geschlechterverhältnisse häufiger thematisiert: etwa als Frage nach einer feministischen Staatstheorie (151/2008), als Element der Spannung zwischen Totalität und Vielfalt in bürgerlichen Gesellschaften (165/2011) oder als mögliche Vereinnahmung feministischer Kritik durch den Mainstream (167/2012). Auf einer grundsätzlichen Ebene wollen wir uns im nächsten Jahr mit dem Thema auseinander setzen, für 2014 ist ein Heft mit dem Schwerpunkt Materialistischer Feminismus geplant (174/2014).

Weitere Themen, die bisher eher Zaungäste bei den bisherigen Theoriedebatten in der PROKLA waren, fanden in den letzten Jahren verstärkt Aufmerksamkeit: Ethnisierung und Ökonomie (PROKLA 12/2000), Migration (140/2005) oder Postkoloniale Studien (158/2010) in ihrem Stellenwert für kritische Sozialwissenschaften. Schließlich haben Überlegungen zur Historisierung des Kapitalismus vermehrt Eingang gefunden, so dass es nicht mehr, wie es früher häufig der Fall war, in erster Linie um grundsätzliche Gesetzmäßigkeiten der kapitalistische Produktionsweise ging. Wiederholt wurde der Regulationsansatz diskutiert, in dem die begrenzte Perspektive der Produktion verlassen und nach Zusammenhängen zwischen deren Normen, denjenigen des Konsums und der gesellschaftlichen Regulierung in verschiedenen Perioden des Kapitalismus gefragt wird, so in PROKLA 58/1985, 72/1988, 100/1995, 113/1998. Nachlesen kann man die meisten der erwähnten Artikel und Hefte auf unserer Website www. prokla.de, wo unter Archiv sämtliche Hefte bis auf die letzten zwei Jahrgänge zum (kostenlosen) Download bereit stehen.

Wie diese kurze Rückschau deutlich machte, hat sich die PROKLA schon lange aus dem eng abgesteckten Feld einer strikt auf Ökonomie und Kapitalherrschaft begrenzten Analyse gelöst. Bereits in den vorigen Heften zur Gesellschaftstheorie wurde betont, dass der Anspruch der Marxschen Untersuchung von Ware, Geld und Kapital darin, die "Anatomie der bürgerlichen Gesellschaft" offen zu legen, einer Gesellschaft also, die nicht in der Ökonomie aufgeht, selbst wenn diese die dominante Struktur darstellt. Um noch einmal auf Wagner zurück zu kommen: Wenn Siegfried den Drachen Fafner, den Bewacher des verhängnisvollen Goldschatzes, tötet, so erscheint er zunächst als strahlender Held. Aber mit dem Besitz des eroberten Rings handelt er sich letztlich nur maßloses Unglück ein. Das Kapital ist nicht einfach nur ein Drachen, den es - etwa, indem bürgerliche Eigentumsformen aufgehoben werden - zu besiegen gilt, vielmehr sind alle Verhältnisse der Ausbeutung von Menschen und Natur und der Entwürdigung von Menschen zu überwinden.

Einige Artikel des vorliegenden Heftes schließen an Beiträge in den vorausgegangenen Heften zur Gesellschaftstheorie an, vor allem aber soll es hier um Auseinandersetzungen mit grundlegenden theoretischen Konzepten gehen. Alex Demirovic untersucht das Verhältnis von marxistisch inspirierten Zeitdiagnosen, wie wir sie bei Adorno finden, und der Analyse von Kräfteverhältnissen in der Tradition von 
Eine lange Zeit eher schattenhafte Existenz führte auch das Thema Geschlechterverhältnisse, das zunächst, wenn überhaupt, im Zusammenhang mit Frauenarbeit diskutiert wurde, so in einzelnen Aufsätzen als „Arbeit der Mütter“ (PROKLA 22/1976) oder als „Hausarbeit“ (33/1978), schließlich als Heftschwerpunkt Frauen in der Ökonomie (93/1993), wo auch erstmals der Ansatz der sozialen Konstruktionen von Weiblichkeit und Männlichkeit zum Thema wurde. In den letzten Jahren wurden Geschlechterverhältnisse häufiger thematisiert: etwa als Frage nach einer feministischen Staatstheorie (151/2008), als Element der Spannung zwischen Totalität und Vielfalt in bürgerlichen Gesellschaften (165/2011) oder als mögliche Vereinnahmung feministischer Kritik durch den Mainstream (167/2012). Auf einer grundsätzlichen Ebene wollen wir uns im nächsten Jahr mit dem Thema auseinander setzen, für 2014 ist ein Heft mit dem Schwerpunkt Materialistischer Feminismus geplant (174/2014).

Weitere Themen, die bisher eher Zaungäste bei den bisherigen Theoriedebatten in der PROKLA waren, fanden in den letzten Jahren verstärkt Aufmerksamkeit: Ethnisierung und Ökonomie (PROKLA 12/2000), Migration (140/2005) oder Postkoloniale Studien (158/2010) in ihrem Stellenwert für kritische Sozialwissenschaften. Schließlich haben Überlegungen zur Historisierung des Kapitalismus vermehrt Eingang gefunden, so dass es nicht mehr, wie es früher häufig der Fall war, in erster Linie um grundsätzliche Gesetzmäßigkeiten der kapitalistische Produktionsweise ging. Wiederholt wurde der Regulationsansatz diskutiert, in dem die begrenzte Perspektive der Produktion verlassen und nach Zusammenhängen zwischen deren Normen, denjenigen des Konsums und der gesellschaftlichen Regulierung in verschiedenen Perioden des Kapitalismus gefragt wird, so in PROKLA 58/1985, 72/1988, 100/1995, 113/1998. Nachlesen kann man die meisten der erwähnten Artikel und Hefte auf unserer Website www. prokla.de, wo unter Archiv sämtliche Hefte bis auf die letzten zwei Jahrgänge zum (kostenlosen) Download bereit stehen.

Wie diese kurze Rückschau deutlich machte, hat sich die PROKLA schon lange aus dem eng abgesteckten Feld einer strikt auf Ökonomie und Kapitalherrschaft begrenzten Analyse gelöst. Bereits in den vorigen Heften zur Gesellschaftstheorie wurde betont, dass der Anspruch der Marxschen Untersuchung von Ware, Geld und Kapital darin, die "Anatomie der bürgerlichen Gesellschaft" offen zu legen, einer Gesellschaft also, die nicht in der Ökonomie aufgeht, selbst wenn diese die dominante Struktur darstellt. Um noch einmal auf Wagner zurück zu kommen: Wenn Siegfried den Drachen Fafner, den Bewacher des verhängnisvollen Goldschatzes, tötet, so erscheint er zunächst als strahlender Held. Aber mit dem Besitz des eroberten Rings handelt er sich letztlich nur maßloses Unglück ein. Das Kapital ist nicht einfach nur ein Drachen, den es - etwa, indem bürgerliche Eigentumsformen aufgehoben werden - zu besiegen gilt, vielmehr sind alle Verhältnisse der Ausbeutung von Menschen und Natur und der Entwürdigung von Menschen zu überwinden.

Einige Artikel des vorliegenden Heftes schließen an Beiträge in den vorausgegangenen Heften zur Gesellschaftstheorie an, vor allem aber soll es hier um Auseinandersetzungen mit grundlegenden theoretischen Konzepten gehen. Alex Demirovic untersucht das Verhältnis von marxistisch inspirierten Zeitdiagnosen, wie wir sie bei Adorno finden, und der Analyse von Kräfteverhältnissen in der Tradition von 
Gramsci und Poulantzas und fragt, inwiefern die Beiträge von Slavoj Žižek dabei helfen können, diese unterschiedlichen Ansätze gesellschaftstheoretisch zu integrieren. Etienne Schneider knüpft an Hanna Meißners Beitrag in PROKLA 165/2011 an, in dem Totalität und Vielfalt aus feministischer Sicht thematisiert wurden, und diskutiert in seinem Beitrag unterschiedliche Kritiken am Intersektionalitätskonzept. Vor diesem Hintergrund wird der Frage nachgegangen, inwiefern Überlegungen aus der marxistischen Gesellschaftstheorie für das Verständnis verschiedener sozialer Herrschaftsverhältnisse und Widersprüche fruchtbar gemacht werden können. Eine schon ältere Kritik von John Bellamy Foster aus PROKLA 76/1989 am FordismusBegriff führt Dorothea Schmidt fort, indem sie fragt, wie sich der gängige Bezug auf Henry Fords Modell der Massenproduktion von Automobilen vor und nach 1914 zur Realität der Fordschen Fabriken und zu der behaupteten Fordismus-Periode in der frühen Bundesrepublik bis Mitte der 1970er Jahre verhält. Damit wird einer der wichtigen Bausteine des RegulationsKonzepts auf den Prüfstand gestellt. Auf eine wichtige Blindstelle im zuletzt vieldiskutierten „Varieties of Capitalism“-Ansatz machen Stefan Beck und Christoph Scherrer aufmerksam: die Finanzialisierung der Ökonomie. Sie zeigen auf, dass nicht nur die Gegenüberstellung eines bankbasierten und eines marktbasierten Finanzsystems unzureichend ist, vor allem gerät bei den „Varieties of Capitalism“-Analysen aus dem Blick, dass der Finanzsektor selbst ein profitgesteuerter Sektor ist und nicht bloß ein Dienstleister für Unternehmen. Das Verhältnis von Karl Marx und Pierre Bourdieu in Bezug auf die Kritik der herrschenden Ökonomie wird von Peter Streckeisen untersucht. Trotz der nicht zu leugnenden grundsätzlichen Differenzen zwischen beiden Ansätzen, sieht Streckeisen Möglichkeiten, wie sich die Marxsche Analyse sozialer Formen und Bourdieus Theorie der Praxis in bestimmten Feldern sinnvoll kombinieren lassen. Ein Artikel der Ökonomen Reinhart und Rogoff machte gleich zweimal Furore: zum einen weil er empirisch zu beweisen schien, dass eine Staatsverschuldung ab 90 Prozent des BIP zu weniger Wachstum führt, was gerne als zusätzliche Rechtfertigung von Austeritätspolitiken benutzt wurde, und zum anderen als enthüllt wurde, wie schlampig der Umgang mit den empirischen Daten in dem Papier tatsächlich war. Hanno Pabl diskutiert anhand der weitreichenden Rezeption dieses Artikels die inner- und außerdisziplinäre Produktion, Zirkulation und Resonanz von „Wahrheiten“ der Mainstream-Ökonomie.

Außerhalb des Schwerpunkts erinnert Urs Müller Plantenberg an einen anderen 11. September: den von den USA unterstützten Militärputsch gegen den gewählten chilenischen Präsidenten Salvador Allende am 11. September 1973 und die anschließende Militärdiktatur. Herbert Panzer kritisiert schließlich den Aufsatz über Finanzdominierte Akkumulation von Alex Demirović und Thomas Sablowski in PROKLA 166/2012. Thomas Sablowski erwidert auf diese Kritik.

\section{Literatur}

Borchmeyer, Dieter (2002): Richard Wagner, Ahasvers Wandlungen, Frankfurt/M.

Der Bayreuther Jahrhundertring, www.delicatessen.org/der-ring-des-nibelungen/background.html, 24. 7.2013.

Holland, Dietmar (1990): Der Ring des Nibelungen, in: Csampai, Attila/Holland, Dietmar: Opernführer, Hamburg: 533-542. Jäger, Michael (2013): Radikal und sinnlich, in: Der Freitag, 28.3. 